\title{
EL CASO UBER EN URUGUAY Y LA VIGENCIA DE LA SUBORDINACIÓN COMO ELEMENTO CENTRAL EN LA DETERMINACIÓN DE LA LABORALIDAD DEL VÍNCULO*
}

\author{
THE UBER CASE IN URUGUAY AND THE CURRENCY \\ OF THE LEGAL SUBORDINATION AS A CENTRAL \\ ELEMENT IN DETERMINING THE LABOUR NATURE \\ OF A RELATIONSHIP
}

\author{
LE CAS UBER EN URUGUAY ET LA MONNAIE \\ DE LA SUBORDINATION JURIDIQUE COMME ÉLÉMENT \\ CENTRAL POUR LA DÉTERMINATION DE LA NATURE \\ DE TRAVAIL D'UNE RELATION
}

Andrea RODRÍGUEZ YABEN**

\begin{abstract}
RESUMEN: Gracias a las nuevas tecnologías se han desarrollado novedosos modelos de negocios y han aparecido nuevas formas de trabajo. La particularidad que es común a la mayoría es que el prestador del servicio se vincula en forma autónoma, aunque —en la mayoría de los casosexistan elementos o indicios manifiestos de que esa no es la verdadera naturaleza de la relación. A efectos de resolver esta cuestión, las distintas tradiciones jurisprudenciales han presentado elementos variados para identificar la naturaleza laboral o autónoma del vínculo; algunas - la mayoría - moviéndose dentro de la noción clásica de dependencia y subordinación jurídica y sus elementos característicos; y otras, dentro de una noción más amplia que no se agota en la existencia de subordinación y toman en consideración otros elementos que no son típicamente laborales. Utilizando como referencia el reciente pronunciamiento de la
\end{abstract}

* Recibido el 4 de noviembre de 2020 y aceptado para su publicación el 15 de marzo de 2021.

** Abogada egresada de la Universidad de la República (Uruguay). Diplomada en derecho laboral colectivo en la Universidad Diego Portales (Chile). Magister (c) en Derecho del Trabajo y de la Seguridad Social en la Universidad de Talca (Chile). 
justicia uruguaya vinculado a la temática en cuestión, en el presente trabajo pretenderemos identificar que sin perjuicio de que pueden existir distintos estándares de laboralidad (entendiendo por éstos, un conjunto omnicomprensivo de elementos, criterios o indicios utilizados para tomar la decisión), la subordinación jurídica sigue siendo el elemento central para determinar la laboralidad del vínculo.

Palabras clave: plataformas digitales, trabajo autónomo, subordinación, indicios de laboralidad.

ABSTRACT: Due to new technologies, new business models have been developed and new ways of working have appeared. The peculiarity that is common to most of these new ways of providing work is that the service provider is linked to the company as a self-employed worker, although — in most cases - there are clear indications that this is not the true nature of the relationship. In order to resolve this problem, the different jurisprudential traditions have presented varied elements to identify whether the nature of the relationship is labour or not. Most have applied the classic notion of legal dependence and subordination and its characterizing elements; and others have opted for a broader notion that is not exhausted by the existence of subordination and takes into consideration other elements that are not typically labor. Taking as a reference the recent pronouncement of the Uruguayan justice regarding the issue, in this paper we intend to identify that without prejudice to the fact that there may be different labor standards (understanding by these, an all-encompassing set of elements, criteria or indications used to take the decision), legal subordination continues to be the central element in determining the employment status of the relationship.

Keywords: digital platforms, self-employment, legal subordination, indications of labour relationship.

RÉSUMÉ: Grâce aux nouvelles technologies, de nouveaux business models ont été développés et de nouvelles façons de travailler sont apparues. La particularité qui est commune à la plupart est que le fournisseur de services est lié de manière autonome, bien que — dans la plupart des cas - il existe des éléments ou des indications évidentes que ce n'est pas la vraie nature de la relation. Pour résoudre ce problème, les différentes traditions jurisprudentielles ont présenté divers éléments pour identifier la nature de travail ou d'autonomie du lien, certains - la majorité_ s'inscrivant dans la notion classique de dépendance et de subordination juridiques et ses éléments caractéristiques; et 
d'autres, dans une notion plus large qui n'est pas épuisée par l'existence de la subordination et prend en considération d'autres éléments qui ne sont pas typiquement du travail. En prenant comme référence le récent prononcé de la justice uruguayenne lié à la question en question, dans ce travail, nous essaierons d'identifier cela sans préjudice du fait qu'il peut y avoir des normes du travail différentes (comprendre par cellesci, un ensemble complet d'éléments, de critères ou d'indications prendre la décision), la subordination juridique continue d'être l'élément central dans la détermination du statut d'emploi du lien.

Mots-clés: plateformes numériques, travail indépendant, subordination, signes d'emploi.

SUMARIO: I. El trabajo en el capitalismo de plataformas como punto de partida. II. El rol de la subcontratación en la calificación jurídica de la relación entre chofer y la empresa de plataforma. III. Más allá de la subordinación. IV. Reflexiones finales.

\section{EL TRABAJO EN EL CAPITALISMO DE PLATAFORMAS COMO PUNTO DE PARTIDA}

1 sistimos a una época de profundos cambios en nuestras vidas, modigitales y la irrupción de la economía digital. Si bien este escenario se ha denominado de diversas maneras con la finalidad de ilustrar sus virtudes o interpelar los riesgos y peligros que lo acompañan (economía disruptiva, cuarta revolución industrial, gig economy, economía colaborativa, economía on-demand, etc.), hay una realidad de fondo que se mantiene sin cambio, y es que seguimos viviendo en una sociedad capitalista en la cual la competencia y la búsqueda de ganancias proporcionan los parámetros de acción. La especialidad que el capitalismo del siglo XXI presenta es su materia prima: los datos; ya que, precisamente, la acción de extracción y análisis de esos datos es la fuente de creación de valor e ingresos.

En este contexto es que emergen las plataformas digitales, ya que irrumpen como novedosos modelos de negocios que, además de proporcionar enormes cantidades de información, cuentan con la capacidad para almacenarla y analizarla. 
Si bien esta nueva realidad adopta múltiples formas, de acuerdo al tipo de prestaciones que se brinda al usuario; ${ }^{1}$ las que están generando que se debata acerca de la "huida" del derecho del trabajo ${ }^{2}$ son aquellas que importan un modelo de negocios que opera sin activos, salvo la propiedad del software y el análisis de los datos. Se trata de plataformas que "operan a través de un modelo hipertercerizado, en el que los trabajadores están deslocalizados, el capital fijo, los costos de mantenimiento y el training están deslocalizados. Todo lo que queda es el mínimo extractivo básico —el control de la plataforma que permite ganar una renta monopólica-". ${ }^{3}$ Este es precisamente el modelo de negocio de Uber, empresa que resulta demandada en el caso objeto del presente análisis.

La forma en que se organiza el trabajo incluye trabajadores sujetos a formas de trabajo flexible y condicionados por la demanda de servicios, en donde la empresa intermediaria tiene el gobierno absoluto sobre la forma en que se desarrolla la actividad objeto del negocio. Estas formas de trabajo fragmentadas, informales y basadas en tareas se han ampliado enormemente, al punto de que asistimos a un cambio desde el modelo de producción fordista a un modelo de producción robotizada, flexible y diversificada que organiza y ordena el trabajo en forma distinta, de modo tal que el poder empresarial se ejerce de manera diferente, por tanto

...las viejas dimensiones jerárquicas y piramidales de la empresa entran en crisis, con los principios tayloristas y el sistema fordista de producción, para

1 En este sentido, Srnicek realiza una división analítica y distingue entre "plataformas publicitarias" como Google o Facebook que extraen información de los usuarios para vender espacio publicitario; "plataformas de la nube" como Amazon Web Services que son propietarias del hardware y del software de negocios que dependen de lo digital y que los rentan de acuerdo con las necesidades; "plataformas industriales" como Siemens, que producen el hardware y software que se necesita para transformar la manufactura tradicional en procesos conectados por Internet que bajan los costos de producción y transforman bienes en servicios; "plataformas de productos" como Spotify que generan ganancias mediante el uso de otras plataformas para transformar un bien tradicional en un servicio y cobrar por ellos un alquiler o una tasa de suscripción; y finalmente están las "plataformas austeras" como Uber o Airbnb que intentan reducir a un mínimo los activos de los que son propietarias y obtener ganancias mediante la mayor reducción de costos posible. Srnicek, Nick, Capitalismo de plataformas, Buenos Aires, Caja Negra, 2018, pp. 51-83.

2 Baylos, Antonio, "La huida del derecho del trabajo: tendencias y límites de la deslaboralización”, en Alarcón, M. y Mirón, M., El trabajo ante el cambio de siglo: un tratamiento multidisciplinar, Marcial Pons, 2000, p. 68.

3 Srnicek, Nick, op. cit., p. 72. 
dar lugar a procesos de control productivo más horizontales y más heterodirigidos en los que el trabajador goza de mayor autonomía en la ejecución de su trabajo, que hace que formalmente haya un cierto relajamiento de las prácticas de dependencia. ${ }^{4}$

Así, los debates acerca del capitalismo de plataformas y su impacto en el espacio laboral parecen remitirnos a los albores del siglo XIX y, en particular, a la viva controversia sobre la calificación de la laboralidad del vínculo de quienes prestan servicios para estas empresas digitales. Desde luego, resulta novedoso el uso de la tecnología y el diseño del negocio, no así el hecho de que, ante una anomalía en la forma típica de prestaciones del trabajo en donde se opta por la contratación de autónomos, se discuta sobre si esta es la verdadera naturaleza de ésta.

\section{EL ROL DE LA SUBCONTRATACIÓN EN LA CALIFICACIÓN JURÍDICA DE LA RELACIÓN ENTRE EL CHOFER Y LA EMPRESA DE PLATAFORMA}

En la actualidad, la mayoría de las tradiciones jurídicas no proporcionan una solución legal expresa o fórmula clara para determinar si quien presta servicio inmerso en este contexto debe clasificarse como trabajador dependiente o autónomo, así que la resolución de esta ambigüedad, cuando se contrata, queda a criterio de la empresa, la que resuelve ir por la autonomía del vínculo con el prestador del servicio a través de fórmulas civiles o mercantiles.

¿Por qué el trabajo autónomo como modalidad para vehicular este modelo de negocio? La respuesta a esta interrogante está en los propios caracteres de éste: por un lado, la flexibilidad en la disposición de la mano de obra, rasgo básico para atender la demanda; por otro, los amplios espacios dejados a la autonomía de las partes, lo que trasunta es una escasa protección para quienes prestan servicios; y finalmente, la reducción de los costos para la empresa.

No obstante, en los hechos se ha demostrado que la demanda impredecible de los consumidores hace que la flexibilidad sea ilusoria, la recopilación de datos invasiva, hasta el punto de afectar la privacidad de los usuarios, ${ }^{5}$ y el control ya no resulta ejercido en forma directa (o más bien física) sino que

4 Rodríguez-Piñero y Bravo Ferrer, Miguel, "Contrato de trabajo y autonomía del trabajador", en varios autores, Trabajo subordinado y trabajo autónomo, Tecnos, 1999, p. 37.

5 Prassl, Jeremias, Humans as a Service, Oxford University Press, 2018, p. 27. 
es delegado a una aplicación, la que resulta ser la herramienta principal de administración, dirección y control del negocio. ${ }^{6}$ Así, las nuevas tecnologías aplicadas al trabajo en plataformas, si bien permiten construir una relación mediada por la distancia física entre la empresa y el trabajador (bajo la narrativa de la autonomía en la prestación del servicio), no impiden en ningún sentido la formación de una relación de trabajo con base en la presencia de control y dirección, por tanto, desde el punto de vista jurídico laboral, la cuestión que se presenta es la de tratar de dilucidar si la relación que surge a través de estas plataformas digitales con los prestadores de servicios, corresponde a una relación de las protegidas por el derecho del trabajo.

Este es precisamente el problema jurídico planteado por el trabajador, y el objeto de las decisiones judiciales que se analizan. ${ }^{7}$

Así, la sentencia de primera instancia señala que se discute

...la naturaleza del vínculo trabado entre las partes, pues mientras el promotor aduce la existencia de un vínculo de trabajo subordinado entre las partes y las califica como de conjunto económico. La demandada alega la existencia de un vínculo de carácter comercial entre el reclamante y Uber, siendo el primero un prestador de servicios de transporte independiente, autónomo y Uber es una empresa de tecnología, no de transporte, por lo cual mal podría ser empleadora.

En idéntico sentido, el Tribunal de Apelaciones precisa que se trata de un problema de "calificación jurídica del vínculo en debate".

A efectos de resolver la controversia, ambas instancias edifican su razonamiento con base en la noción de subordinación, y especialmente a la Recomendación núm. 198 de la Organización Internacional del Trabajo (OIT) como premisa normativa. Si bien existe consenso en cuanto al material normativo aplicable, no es así en cuanto al alcance e interpretación de éste, ya que mientras en primera instancia se consigna a la subordinación como "elemento infaltable para determinar o no la existencia de la relación laboral", el Tribunal de Apelaciones plantea una superación de esta noción a efectos de determinar

6 Ivanova, Mirela et al., "The App as a Boss? Control and Autonomy in Application-Based Management”, Arbeit | Grenze | Fluss - Work in Progress Interdisziplinärer Arbeitsforschung, $\mathrm{N}^{\circ} 2$, 2018, pp. 7-8.

7 “Queimada, Esteban c/Uber Technologies Uruguay S.A. y otro", IUE 2-3894/2019. Entendiéndose en primera instancia el Juzgado Letrado del Trabajo de la Capital de $6^{\circ}$ Turno (11.11.2019) y en segunda el Tribunal de Apelaciones de Trabajo de 1er. Turno (3.6.2020). 
la laboralidad del vínculo al señalar que "tal característica puede estar o no estar". Este aspecto resulta central, ya que interpretaciones divergentes pueden dar lugar a respuestas distintas a la cuestión que ha de resolverse, no obstante, este no fue el caso, ya que en ambas instancias se decidió a favor de la laboralidad del vínculo, poniendo el énfasis del análisis jurídico en la presencia fáctica de subordinación y dependencia del trabajador que se desempeñaba como chofer.

Evidentemente el juez, en tanto aplicador de normas, aunque esté vinculado por las directrices establecidas en las mismas, tiene libertad para seguir distintos métodos de interpretación, exigiéndose —a efectos de la justificación en la premisa adoptada - solidez en la argumentación, la identificación de los criterios hermenéuticos utilizados y un razonamiento enmarcado en el material jurídico vigente. $^{8}$ Asimismo, dicha construcción debe tener conexión con la decisión final, ya que de lo contrario la elaboración resulta estéril. Tal como relevaremos, este estándar no resulta ser superado en el razonamiento del Tribunal de Apelaciones en cuanto a la superación de la subordinación que propone.

En mérito a lo referido, nos proponemos analizar las decisiones judiciales y las razones que determinaron las mismas. Para ello, dejaremos a un lado la lógica formal ${ }^{9}$ como instrumento para explicar el proceso decisorio para centrarnos en cuestiones materiales, es decir, en los motivos sobresalientes que condicionaron el contenido de las decisiones y los criterios empleados por el juez de instancia y por el Tribunal.

\section{La subordinación jurídica como elemento normativo esencial en la operación de calificación de las relaciones protegidas por el derecho del trabajo}

Actualmente existe consenso en la doctrina uruguaya ${ }^{10}$ en identificar a la subordinación jurídica como el criterio calificador de las relaciones de trabajo

8 Al respecto, véase Segura, Manuel, La racionalidad juridica, Madrid, Tecnos, 1998, pp. 73-114.

9 Entendiendo por ésta el modelo conforme al cual "los sujetos encargados de realizar la tarea de aplicación desarrollan una labor puramente cognoscitiva que se reduce a la realización de una serie de operaciones lógicas. La aplicación de la ley consiste básicamente en subsumir los hechos que se presentan ante el juez bajo la norma legal y de un modo silogístico extraer las consecuencias previstas en la norma”. Ibidem, p. 75.

10 A modo de ejemplo, Américo Plá Rodríguez afirma que "La subordinación es el criterio distintivo del contrato de trabajo y, por consiguiente, la llave de paso para la aplicación del de- 
protegidas por el sistema normativo laboral, y ello por cuanto se encuentra dotado de mejores cualidades a efectos de cumplir la finalidad de calificación, ya que "a lo largo de su amplia andadura histórica, la noción de dependencia ha mostrado poseer una extraordinaria capacidad de adaptación a la diversidad de perfiles bajo los cuales comparece en la realidad el fenómeno económico y social de trabajo asalariado". ${ }^{11}$

Por su parte, la jurisprudencia ${ }^{12}$ ha acompañado esta posición, lo que en su momento resulta plasmado en las decisiones que se comentan, aunque con algunas diferencias en cuanto a su centralidad como figura normativa para determinar la naturaleza laboral de la relación.

Así, la sentencia de primera instancia precisa a la subordinación y la remuneración como elementos imprescindibles en la calificación jurídica de la relación como de naturaleza laboral, previniendo que "no basta con poner sus energías al servicio de otro para conformar la relación laboral".

En su oportunidad, la sentencia del Tribunal de Apelaciones —-basándose en una interpretación de lo establecido en la Recomendación núm. 198 de la OIT— señala que, si bien el instrumento internacional

...considera la subordinación, la desfocaliza como el aspecto principal y excluyente en la solución del conflicto, y lo focaliza en un elenco mucho más abierto que de todos modos lo incluye. Pero en pie de igualdad en cuanto a su trascendencia. Por lo que admite la relación de trabajo aun sin la subordinación en su concepto clásico, si se detectan uno o varios de los restantes indicadores.

Si bien dicho párrafo resulta impreciso en cuanto a la determinación de si es la subordinación o no el elemento fundamental para calificar una relación como laboral, seguidamente lo clarifica, ya que expresamente señala que

recho del trabajo". Al respecto, véase Rivas, Daniel, La subordinación: criterio distintivo del contrato de trabajo, Montevideo, Fundación de Cultura Universitaria, 2001, p. 17.

11 Sanguinetti, Wilfredo, "La dependencia y las nuevas realidades económicas y sociales: ¿un criterio en crisis?”, Temas Laborales. Revista Andaluza de Trabajo y Bienestar Social, núm. 40, 1996, p. 53.

12 La mayoría de las tradiciones jurisprudenciales, a efectos de determinar la naturaleza del vínculo, analizan la existencia de indicios de subordinación y dependencia del que presta el servicio a favor de la empresa digital. A modo de ejemplo, puede verse el exhaustivo análisis de varias tradiciones jurisprudenciales realizado en University of Oxford, "The Employment Status of Uber Drivers. A Comparative Report Prepared for the Social Law Project, University of the Western Cape"; disponible en https://ohrh.law.ox.ac.uk/wordpress/wp-content/ uploads/2017/10/OPBP-Uber-Project-Final-pdf. 
...la subordinación o dependencia deja de ser una condición necesaria y excluyente, para reposicionarse en una condición suficiente. Ello se infiere de la interpretación del texto de la Recomendación cuando, en sede de las políticas a adoptar por los Estados sugiere la subordinación o dependencia a título de ejemplo. Ello autoriza a inferir que tal característica puede estar o no estar, y si puede estar o no estar también podría adoptar una resolución intermedia consistente en su reformulación.

A nuestro entender, el razonamiento incurre en una confusión entre tipo normativo y manifestaciones de aquél; en otras palabras, entre subordinación e indicios, ya que del propio texto de la sentencia se infiere que se otorga a los indicios un valor propio y distinto de la noción de subordinación, aunque equivalente, cuando en rigor no lo son.

\section{2. ¿Qué justifica que sea la subordinación el elemento fundamental para determinar la laboralidad del vinculo?}

Básicamente, la existencia de poder. En efecto, el poder es "un punto clave en el campo de las relaciones laborales" ya que las mismas "están centradas en los procesos de influencia y control sobre el trabajo". ${ }^{13}$ En efecto, la relación de trabajo se construye como un espacio en donde existe una desigual distribución de poder, siendo los trabajadores los que se encuentran en situación de sujeción respecto del otro, en tanto "la empresa es quien decide si habrá trabajo o no, y la gerencia quien disfruta el derecho a decidir cómo se va a organizar el trabajo", 14 en este sentido, "individualmente considerado, el empleado [...] no detenta normalmente poder social alguno, puesto que, en cuanto individuo, sólo muy excepcionalmente posee poder de negociación". ${ }^{15}$

En este sentido, la protección del derecho del trabajo se justifica en aquellos casos en los cuales el trabajador se encuentra en una situación de contracción a su libertad, en el sentido de pérdida de autonomía o autodeterminación en la ejecución de la tarea encomendada, poniéndose bajo el control y la dirección de otro (empleador). Ahora bien, para hacer operativa esa protección

13 Kirkbride, Paul, "El poder", en varios autores, Relaciones laborales. La psicología de la influencia y el control en el trabajo, Madrid, Ministerio del Trabajo y Seguridad Social, 1994, p. 121.

14 Kennoy, Tom, "La creación de mecanismos de control", en varios autores, Relaciones laborales..., cit., p. 159.

15 Kahn-Freund, Otto, Trabajo y derecho, Madrid, Ministerio del Trabajo y Seguridad Social, 1987 , p. 59. 
...se utiliza un tipo abierto - la subordinación - cuyo contenido se refiere a la dirección y control que un tercero —el empleador- ejerce sobre ese trabajador. La forma de la existencia de esa subordinación es determinar para el caso concreto la presencia relevante de manifestaciones — denominados indicios - de esa pérdida de libertad en la forma en que se presta o se ejecuta el trabajo convenido. ${ }^{16}$

Así, mientras que la subordinación se construye como el elemento que permitirá determinar quiénes serán protegidos por las normas del derecho del trabajo — los trabajadores en dependencia jurídica-, los indicios permitirán evidenciar en el caso concreto la presencia relevante de manifestaciones de esa pérdida de autonomía.

La interpretación sostenida por el Tribunal de Apelaciones se aparta de esta construcción, y de la posición histórica en Uruguay respecto a la noción de subordinación como criterio central de distribución de la protección del derecho del trabajo, y postula una superación o desborde de la misma, pero sin argumentar con claridad cuál es el otro criterio de configuración del derecho del trabajo y cuáles son las razones subyacentes al mismo, a la vez que se pierde de vista que los indicios o indicadores son expresión de algo más, y no tipos normativos autónomos con un valor en sí mismo, por lo que mal pueden constituirse como criterios equivalentes a la subordinación.

A pesar de estas diferencias interpretativas en cuanto a la premisa normativa, el planteamiento del razonamiento judicial en ambas instancias se estructura con base en manifestaciones que expresan el contenido propio de la subordinación, tal como afirma el Tribunal: "la ostensible constricción a la libertad en la ejecución de la tarea y en el beneficio que ella podría reportarle, no habilitan reconstruir un contrato comercial sino una relación de trabajo”. Por tanto, la construcción que apuesta a una superación de esta noción no resulta justificada.

\section{La técnica indiciaria como mecanismo de aplicación del tipo normativo}

Relevada la centralidad de la subordinación como criterio de distribución de la protección laboral, y despejado que el contenido propio de la misma consiste en el control y dirección ejercidos sobre el trabajador, surge la siguiente interrogante: ¿cómo se determina su presencia para el caso concreto?

16 Ugarte, José Luis, "Trabajo en empresas de plataforma: subordinación y más allá”, Revista Chilena de Derecho Privado, Santiago, Universidad Diego Portales, núm. 35, 2020, p. 7. 
Ambos pronunciamientos consideran que dicha operación es a través del haz de indicios postulados en la Recomendación núm. 198 de la OIT como el marco teórico aplicable en Uruguay cuando existe controversia en la calificación de una relación jurídica que compromete trabajo, pues no existe en dicho país expresión normativa alguna que defina la frontera entre el trabajo al amparo del derecho del trabajo y el prestado en forma autónoma, considerando el documento emitido por el organismo internacional como una fuente de derecho.

Así, el protagonismo de la Recomendación mencionada resulta central en ambas decisiones, y opera como una suerte de guía argumentativa en la determinación de las notas caracterizantes de una relación laboral, no obstante hay una diferencia en cuanto al valor jurídico otorgado a dicho instrumento, ya que mientras la sentencia de primera instancia señala que se trata de "una herramienta de interpretación para determinar la posición de cada sujeto en la relación debatida", la sentencia del Tribunal advierte que "debe ser utilizada como modelo normativo para sustituir o complementar la elaboración doctrinaria desarrollada antes de su vigencia", con el fin de "apreciar y calificar los hechos de la realidad en situaciones en las que se compromete trabajo y la naturaleza del vínculo se encuentra en debate".

Si bien la Recomendación mencionada es un elemento útil a considerar por el juez con el fin de edificar el razonamiento jurídico, no puede perderse de vista que dicho instrumento internacional nace como una propuesta ante la restricción de libertad evidenciada en aquellas relaciones de trabajo típicas, por lo que los indicadores propuestos para identificar una relación como de naturaleza laboral tienen que ver con la sujeción física del trabajador en un lugar determinado, es por ello que los indicios que se expresan aluden a instrucciones directas de otra persona, la integración organizacional, un horario determinado, trabajar en el lugar o local de la empresa, suministro de herramientas, materiales y maquinarias por parte del empleador, continuidad, etc.

Como se señaló anteriormente, el desarrollo de las tecnologías, la transformación digital y su integración en novedosos modelos de negocios, han llevado a que surjan nuevas formas de trabajo, en este sentido, el espacio de trabajo se transforma, "se deslocaliza por intermediación tecnológica, se trabaja a distancia y las relaciones se establecen a través de redes. Las redes neuronales, los robots y aplicaciones de inteligencia artificial se convierten en un instrumento fundamental de la producción: procesos enteramente gestionados por las máquinas". ${ }^{17}$

17 Mercader, Jesús, El futuro del trabajo en la era de la digitalización y la robótica, Valencia, Tirant lo Blanch, 2017, p. 31. 
En este sentido, la objeción podría residir en que un apego muy estricto al catálogo de indicadores que allí se mencionan podría importar el desconocimiento de otras manifestaciones que responden a una forma de trabajo que no existía (o al menos no en la relevancia que tiene hoy) en la época en que dicho instrumento fue confeccionado. Así, darle un valor normativo que pretenda capturar la esencia de la subordinación como tipo abstracto puede hacer muy rígida la operación de calificación y omitir elementos que sí dan cuenta de que el trabajador está en una situación de sujeción pero que no se relevan por no encontrarse previstos en dicho haz indiciario.

Por ejemplo, el trabajador ya no se encuentra desempeñando la actividad en un espacio determinado, la intensidad y ritmo productivo no requieren integrar laboralmente al trabajador a la organización, la jornada no es continua ni la remuneración necesariamente periódica o exclusivamente la única fuente de ingresos del prestador del servicio, las herramientas no son proporcionadas por el empleador, etc.

Si bien la operación de calificación se logra a través de la técnica indiciaria, es decir, a través del análisis de las manifestaciones presentes en el caso concreto que den cuenta del ejercicio de dirección y control por parte del empleador, esto va a depender de la realidad productiva del momento en cuanto a formas de expresarse el poder en la relación de trabajo. Al respecto, se ha precisado que no existe un conjunto "estructuralmente inherente de indicios en la subordinación, ya que su presencia es contingente para cada caso", por tanto, "no es que, en rigor, existan nuevos indicios que reemplacen o sustituyan a los antiguos sobre la misma base o contenido, sino que hay indicios propios de otra forma de control/dirección que comienza a extenderse en el trabajo asalariado: el control virtual o algorítmico". ${ }^{18}$

En este sentido, el análisis del aplicador del derecho debería enfocarse en el examen específico de la forma en que se presta el servicio o las tareas encomendadas, para evidenciar si de la misma surge que no existe autonomía del trabajador en su ejecución, ya que, por el contrario, un tercero ordena, controla y disciplina el proceso productivo, lo que da mérito a la tutela laboral. En este sentido, los indicadores pueden ser múltiples y variados dependiendo de la actividad, el tipo de negocio, la organización productiva en la que esté inmerso el trabajador, etc. De allí que pueda afirmarse que el procedimiento de calificación debe estar encaminado al examen de la realidad a la luz de las nociones de dirección y control, y no a la identificación o subsunción a indicios o indicadores señalados en forma general y abstracta.

18 Ugarte, José Luis, op. cit., pp. 8-9. 


\section{Manifestaciones de la sujeción del trabajador al poder del empleador}

Ambas instancias fueron coincidentes en inclinar la balanza hacia la laboralidad del vínculo entre el chofer y la empresa Uber, otorgándole un valor significativo al grado de dirección y control que se ejerce sobre el trabajo prestado, y, por tanto, al sometimiento del trabajador al círculo rector, disciplinario y organizativo de la empresa digital. Asimismo, es coincidente el rol central que se otorga al principio de primacía de la realidad como criterio interpretativo.

En este contexto, exponiéndose la prevención de que los indicadores que se describen no pueden apreciarse aisladamente de los restantes, sino en su conjunto y en forma integral, se observan los siguientes indicios como relevantes.

\section{A. Uber se benefició principalmente con el trabajo prestado}

Señala el Tribunal de Apelaciones que el trabajo realizado en beneficio de otro cobra una nueva dimensión en la Recomendación, ya que prevé que el beneficio del trabajo puede ser o bien únicamente para otro, o bien "principalmente" para otro. Así, se señala que basta que el sujeto a quien se entrega el trabajo haya asumido el riesgo en forma principal para entenderlo como un indicio de laboralidad. Así, se señala que Uber se hizo cargo de la tecnología — la driver app — así como del diseño y establecimiento de las tarifas y del porcentaje de cada uno en el reparto de lo recaudado. Esto no lo fijó el trabajador, ni éste en negociación con Uber, sino Uber unilateralmente. A tal punto, que como surge de la plataforma fáctica, el conductor aceptaba el viaje sin saber exactamente cuánto recibiría, en tanto que la distancia recorrida y los importes por tiempo únicamente podría ser determinados por la empresa.

\section{B. Integración del trabajador a la organización de Uber independientemente de la naturaleza de la empresa}

Si bien Uber argumentó que lo que proporcionaba en el vínculo jurídico era la tecnología, la realidad es que el conductor efectivamente la utilizó, y además lo hizo en forma gobernada por la empresa, quien era la que recibía las solicitudes de traslado, las asignaba a cada conductor, resolvía unilateralmente los criterios mediante los cuales distribuía tal asignación, 
cobraba directamente la tarifa al usuario y gestionaba el cobro del servicio. En rigor, el trabajador solamente se encargaba de la ejecución del traslado de los pasajeros.

Este aspecto es considerado en las decisiones judiciales. Así, en primera instancia se precisa que "la labor desempeñada por el accionante durante años constituyó un eslabón de la actividad de la demandada por su cuenta y riesgo y parte de su organización”. En su oportunidad, señala el Tribunal que el debate acerca de si Uber es una empresa de transporte o una empresa de tecnología, carece de incidencia, este aspecto podría gravitar luego de haberse concluido en la calificación como relación de trabajo, para poder conocer el salario y las condiciones de trabajo. Lo relevante es que la empresa utilizaba la herramienta tecnológica para organizar su sistema productivo, conectar la demanda de servicios de transporte con la oferta respectiva, y gestionar el cobro del servicio al usuario. En su momento, el trabajador se ocupaba de realizar el trasporte del usuario que Uber le adjudicaba con la sola opción de tomar o dejar la actividad que la empresa disciplinaba. Por lo que se infiere, sin obstáculo, que la labor del trabajador constituye un eslabón del proceso productivo organizado y comandado por Uber, y al que se lo somete y se lo integra, ocupando una función concreta que armoniza con el propósito de Uber, y que sin ella la labor de traslado de pasajeros no existiría como negocio.

\section{Realización del trabajo según instrucciones o bajo el control de otra persona}

Señala el Tribunal de Apelaciones que este indicador que prevé la Recomendación aludida es de "textura abierta en tanto no refiere a la forma de emitir instrucciones ni a su alcance, y tampoco a la entidad del control". Para interpretarlo entonces, en la sentencia de segunda instancia se indagaron dos aspectos conexos: por un lado, si Uber emitió algún tipo de instrucción y si ejerció algún tipo de control; y, por el otro, el grado de libertad en la ejecución de la tarea comprometida por el trabajador.

Referente al último aspecto señalado, se precisa un elemento potente en la argumentación y es que ello debe apreciarse desde la faz de ejecución de la tarea, lo que trae aparejado que no interese como elemento a valorar la intensidad de la libertad para conectarse o no, sino después de conectarse y a partir de que asume la realización del traslado. En definitiva, lo relevante es si el trabajador en la ejecución del transporte de pasajeros podía desenvolverse con libertad en el sentido de autodeterminación, resolviendo cómo 
y para quién hacerlo o si estaba sujeto a las indicaciones de Uber. Tal como apunta acertadamente el Tribunal "Este es el sesgo de la libertad que interesa, por cuanto es la libertad a la hora de la realización de la tarea asumida la que caracteriza al trabajo autónomo”. El autónomo organiza la tarea y la ejecuta siguiendo sus decisiones, sin perjuicio de que el comitente del trabajo controle el resultado. En el caso, el trabajador ejecutaba los traslados conforme las instrucciones que surgen del contrato previstas unilateralmente por la empresa: trasladaba al pasajero que Uber le atribuía, no conocía ni al usuario ni sus datos hasta que Uber se lo comunicaba, no podía contactarse directamente con usuarios, debía trasladarlos en el automóvil registrado que, además, debía mantener en determinadas condiciones de higiene, no podía realizar paradas ni interrupciones en el trayecto, tenía prohibido mostrar cualquier tipo de elemento que lo identificaran con Uber (nombre, colores, uniforme u otra prenda, logotipos, etc.), no podía realizar viajes durante más de ocho horas consecutivas o doce discontinuadas por día. Asimismo, debía realizar la tarea teniendo en cuenta las actualizaciones o modificaciones realizadas "por Uber a su discreción de cuando en cuando" (así lo estipulaba el contrato firmado).

También estaba sujeto a control por parte de la empresa, le examinaba la ruta que realizaba, pudiendo calificarla en caso de entenderla poco eficiente, o si no finalizaba el servicio o si se producía una falla técnica, de hecho, la empresa podía reducir o cancelar la tarifa. Por su parte, Uber elabora un perfil del conductor a partir de las calificaciones de los usuarios y les exige mantener una calificación mínima. Incluso, la empresa insta a los usuarios a notificarle inmediatamente de cualquier incumplimiento o sospecha de incumplimiento o uso o revelación de una ID del conductor.

\section{Poder disciplinario y sancionador}

En el material fáctico, se pone de relieve que si el trabajador tiene una calificación por debajo de aceptable, y el conductor no lo soluciona en el tiempo que Uber le asigna, la empresa podía desactivarle el acceso a la aplicación. Además, el rechazo repetido de peticiones de transporte generaba una experiencia negativa para el conductor y debía cerrar la aplicación. En consecuencia, señala el Tribunal, "Uber dirige y controla toda la actividad del conductor. Incluso responde con actos que perjudican al chofer representando una modalidad de ejercicio del poder sancionador típico de un empleador y no de un contratista comercial". 
Se relevan asimismo otros indicios como la continuidad del trabajador en la empresa (tres años aproximadamente), la retribución periódica, el suministro de herramientas (entendiendo por éstas la driver app y todo su gobierno) y la dependencia económica.

\section{MÁS ALLÁ DE LA SUBORDINACIÓN}

La operación de calificación de laboralidad toma especial relevancia para los jueces, ya que son quienes deben definir si la relación es laboral o no en función de diversos criterios emanados de la ley o la doctrina. Así pues, a efectos de resolver esta dicotomía, las distintas tradiciones jurisprudenciales han presentado elementos variados para identificar la naturaleza laboral o autónoma del vínculo, algunas — la mayoría — moviéndose dentro de la noción de dependencia y subordinación jurídica y sus elementos caracterizantes (tal como en el caso analizado); y otras, dentro de una noción más amplia que no se agota en la existencia de subordinación y toma en consideración otros elementos que no son típicamente laborales. Un ejemplo de ello es el denominado "Test ABC", estándar sostenido en el estado de California (primero por la jurisprudencia y luego recogido en una $l e y^{19}$ ), que pone el acento más allá del control y dirección del trabajador, exigiendo un escrutinio más exigente para descartar la calificación de laboralidad.

El 30 de abril de 2018, la Corte Suprema de California ${ }^{20}$ adoptó por primera vez un estándar para determinar la laboralidad de una relación, el mismo (que resulta ser distinto al utilizado anteriormente por la misma jurisdicción) ${ }^{21}$

19 La Ley denominada "AB-5 Worker status: employees and independent contractors", vigente desde enero de 2020, modifica el Código del Trabajo del estado de California y señala que el "Test $A B C$ " resulta de aplicación para todos los trabajadores salvo excepciones referidas expresamente como algunas ocupaciones o vínculos contractuales específicos. Así, esta norma ordena a las empresas a cumplir con dicho estándar al momento de calificar la naturaleza del vínculo con sus trabajadores, estableciéndose que en caso de que clasifiquen erróneamente a sus trabajadores como autónomos pueden ser sancionados con multas, además de ser objeto de demandas por los trabajadores o por el propio Estado.

20 Dynamex Operations West, Inc. v. Tribunal Superior del Condado de Los Ángeles (Sentencia de fecha 30 de abril de 2018); disponible en http://www.courts.ca.gov/opinions/documents/S222732. PDF.

21 En su decisión, la Corte Suprema dejó a un lado el "Test Borello" tomado como referencia a partir del caso S.G. Borello \& Sons, Inc. v. Departamento de Relaciones Industriales (1989); disponible en https:// law.justia.com/ cases/california/supreme-court/3d/48/341.html. El mismo evaluaba múltiples elementos para determinar si un prestador de servicios era un trabajador 
impone una carga significativamente más alta a las empresas, ya que parte de una presunción de existencia de la relación laboral, que puede superarse si la empresa puede acreditar copulativamente tres elementos: a) que el prestador del servicio esté libre de control y dirección sobre el desempeño de su trabajo; b) que el trabajo proporcionado esté fuera del giro habitual de la empresa; y c) que el trabajador se dedique habitualmente a un oficio, ocupación o negocio en forma independiente de la misma naturaleza que el trabajo realizado.

Uno de los aspectos más destacables es la carga de la prueba que se impone a la empresa, ya que el punto de partida es una presunción de laboralidad, la que, a todas luces, no resultará fácil derribar, salvo que la relación verdaderamente sea autónoma.

Así, a diferencia del estándar clásico analizado antes, los indicios de subordinación y la existencia de control no son los elementos más importantes para determinar si existe una relación de trabajo, sino sólo uno de los tres elementos a considerar. Concretamente, forman parte de la primera interrogante (parte "A") que se hace este estándar: ¿el trabajador presta sus servicios libres de control y dirección por parte de la empresa? A efectos de responderla, se analizan los mismos criterios que los analizados en el estándar anterior, es decir, indicios de subordinación.

La segunda interrogante que debe responderse (parte "B") tiene relación con un aspecto más amplio y que refiere a la organización del negocio: ¿el trabajador realiza un trabajo que está fuera del giro habitual de la empresa? La presente fórmula sugiere que se evalúe si los trabajadores participan realmente en una actividad marginal o si el negocio está siendo utilizado por el empleador para cumplir servicios propios de su giro, y por tanto se utiliza la figura autónoma para evadir las obligaciones salariales, impositivas y de otro tipo. ${ }^{22}$

Finalmente, la última interrogante a plantearse con el propósito de superar esta prueba (parte "C") es: ¿el trabajador desarrolla habitualmente una profesión, ocupación o negocio de la misma naturaleza que el trabajo realizado para la empresa contratante? Para cumplir con esta parte, la empresa debe demostrar que el trabajador desarrolla una actividad de manera independiente por fuera de la empresa a la cual presta servicios, debe ser una actividad

dependiente o autónomo, siendo el principal de ellos el ejercicio de control sobre la forma en que se realiza el trabajo.

22 Deknatel, Anne y Hoff-Downing, Lauren, "ABC on the Books and in the Courts: An Analysis of Recent Independent Contractor and Misclassification Statutes", Univ. of Pennsylvania Journal of Law and Social Change, núm. 53, 2015. 
autónoma estable y duradera que sobrevivirá a la terminación de la relación con la empresa contratante.

Este estándar utilizado a efectos de la calificación del vínculo no se agota en los indicios de subordinación, sino que se pretende ver más allá de esos elementos e incorpora al análisis aspectos vinculados a la forma de organización del negocio y de la naturaleza del servicio en particular, en rigor conjuga elementos típicamente laborales con otros que no lo son. Pero, además, parece comprender a cabalidad el nuevo fenómeno y ampliar la protección del derecho del trabajo a quienes se encuentran prestando servicios para las empresas de plataformas, al partir de una presunción de laboralidad —lo que parecería ser la realidad de estos trabajadores- y trasladar a la empresa la carga de probar la existencia de autonomía en la prestación del servicio.

\section{REFLEXIONES FINALES}

La generalización del trabajo a través de las empresas digitales, y en particular las condiciones en las que se presta el mismo, han iniciado un debate legislativo, doctrinal y jurisprudencial vinculado a la adecuada protección laboral de quien actúa en el negocio como trabajador. Se trata de una situación muy particular, en tanto la "autonomía" o flexibilidad en la cual se encuentra inmerso el trabajador de plataformas viene de la mano de nuevas herramientas de control a disposición de la empresa, lo que nos pone de frente a un conflicto que es del interés de todas las tradiciones jurídicas ordenar.

La cuestión es la siguiente, ¿la relación que surge a través de estas empresas digitales con sus "colaboradores" corresponde a una relación de las protegidas por el derecho del trabajo?

En un escenario como el actual, con falta de orientación política y legal que establezca una dirección determinada, la labor jurisprudencial resulta fundamental. Debido a lo anterior, toma relevancia desmenuzar las fórmulas utilizadas por las distintas tradiciones jurisprudenciales con el fin de determinar la naturaleza del vínculo.

Así, es posible señalar que la mayoría de las tradiciones jurídicas consideran la subordinación como elemento central para determinar la laboralidad del vínculo, dando señales de querer insistir en la senda de flexibilizar el alcance del concepto de subordinación para hacerlo aplicable a esta nueva forma de organizar el trabajo, y no de moverse por fuera del mismo. En general, se estima la laboralidad del vínculo en tanto el trabajador es ajeno a la organi- 
zación, al dominio de la demanda de los usuarios y a la determinación de la tarifa, su ajuste y cambios. En efecto, la prestación del servicio es dirigida y controlada por la empresa de plataformas desde la etapa inicial, a través de la firma de un contrato de adhesión, sin el mínimo poder de negociación para el trabajador, y durante toda la ejecución.

En el caso presentado ante la justicia uruguaya, la estructura argumentativa de las decisiones judiciales se construye justamente a partir de la noción de subordinación, ya que para descartar la calificación realizada por Uber en cuanto a que el trabajador era autónomo, se advierte que gravitan mayoritariamente los indicadores del dominio de la empresa a través del ejercicio constante de dirección y control sobre el trabajador. Así, la ostensible constricción a la libertad del trabajador en la ejecución de la tarea permite ubicar al trabajador dentro del colectivo protegido por el derecho del trabajo. En esta operación de calificación, la técnica de indicios — propia del estándar de la subordinaciónresulta esencial y se ve enriquecida por la concurrencia de nuevos elementos, sin perjuicio de ello, la importancia y centralidad que se da a la Recomendación núm. 198 de la OIT resulta una diferencia ostensible si la comparamos con la construcción argumentativa de decisiones que se han adoptado en otras tradiciones jurídicas.

En definitiva, Uruguay se suma a la lista de países en donde la jurisprudencia se inclina por la condición de asalariado de los trabajadores de plataformas, considerando para su calificación la presencia de subordinación como el elemento normativo esencial en la operación de calificación de las relaciones protegidas por el derecho del trabajo.

\section{REFERENCIAS BIBLIOGRÁFICAS}

BAYLOS, Antonio, "La huida del derecho del trabajo: tendencias y límites de la deslaboralización”, en ALARCÓN, M. y MiRÓN, M., El trabajo ante el cambio de siglo: un tratamiento multidisciplinar, Marcial Pons, 2000.

Deknatel, Anne y HofF-Downing, Lauren, "ABC on the Books and in the Courts: An Analysis of Recent Independent Contractor and Misclassification Statutes", Univ. of Pennsylvania Journal of Law and Social Change, núm. 53, 2015.

Ivanova, Mirela et al., "The App as a Boss? Control and Autonomy in Application-Based Management", Arbeit | Grenze | Fluss-Work in Progress Interdisziplinärer Arbeitsforschung, núm. 2, 2018. 
Kahn-Freund, Otto, Trabajo y derecho, Madrid, Ministerio del Trabajo y Seguridad Social, 1987.

KENNOY, Tom, "La creación de mecanismos de control", en varios autores, Relaciones laborales. La psicología de la influencia y el control en el trabajo, Madrid, Ministerio del Trabajo y Seguridad Social, 1994.

KIRKBRIDE, Paul, "El poder", en varios autores, Relaciones laborales. La psicología de la influencia y el control en el trabajo, Madrid, Ministerio del Trabajo y Seguridad Social, 1994.

MERCADER, Jesús, El futuro del trabajo en la era de la digitalización y la robótica, Valencia, Tirant lo Blanch, 2017.

PRASSL, Jeremias, Humans as a Service, Oxford University Press, 2018.

RIVAS, Daniel, La subordinación: criterio distintivo del contrato de trabajo, Montevideo, Fundación de Cultura Universitaria, 2001.

Rodríguez-PiÑero y Bravo Ferrer, Miguel, "Contrato de trabajo y autonomía del trabajador", en varios autores, Trabajo subordinado y trabajo autónomo, Tecnos, 1999.

SANGUINETTI, Wilfredo, "La dependencia y las nuevas realidades económicas y sociales: ¿un criterio en crisis?”, Temas Laborales. Revista Andaluza de Trabajo y Bienestar Social, núm. 40, 1996.

SEgurA, Manuel, La racionalidad jurídica, Madrid, Tecnos, 1998.

SRniCEK, Nick, Capitalismo de plataformas, Buenos Aires, Caja Negra, 2018.

UGARTE, José Luis, "Trabajo en empresas de plataforma: subordinación y más allá”, Revista Chilena de Derecho Privado, Santiago, Universidad Diego Portales, núm. 35, 2020.

UNIVERSiTy OF OXFORD, “The Employment Status of Uber Drivers. A Comparative Report Prepared for the Social Law Project, University of the Western Cape"; disponible en https://obrh.law.ox.ac.uk/wordpress/wpcontent/ uploads/2017/10/OPBP-Uber-Project-Final-pdf, 2017. 